\section{Prevalence of physical inactivity and associated factors among adolescents from public schools in Uruguaiana, Rio Grande do Sul State, Brazil}

\author{
Prevalência e fatores associados à inatividade física \\ entre adolescentes da rede pública de ensino de \\ Uruguaiana, Rio Grande do Sul, Brasil
}
Prevalencia y factores asociados con la inactividad física en adolescentes de escuelas públicas en Uruguaiana, Rio Grande do Sul, Brasil

Universidade Federal do
Pampa, Uruguaiana, Brasil.
2 Universidade Federal de
Pelotas, Pelotas, Brasil.
Correspondence
G. G. Bergmann
Universidade Federal do
Pampa.
BR 472, Km 592, C.P. 118,
Uruguaiana, RS
97500-970, Brasil.
gabrielgbergmann@gmail.com

This study aimed to identify the prevalence of physical inactivity and associated factors in adolescents, using a cross-sectional design with a sample of 1,455 adolescents (741 females). Data were collected using a questionnaire consisting of socio-demographic, perceptual, and behavioral variables. Physical activity was estimated with the Physical Activity Questionnaire for Older Children and Adolescents. Prevalence of physical inactivity was 68\% (95\%CI: 65.6\%-70.4\%). The following variables remained associated with physical inactivity in the adjusted analysis $(p<0.05)$ : living in an apartment, female gender, older adolescents, lower self-rated physical activity compared to peers, low perception of maternal physical activity, passive commuting to school, non-participation in physical education at school, non-enjoyment of physical education classes, and limited involvement in other types of physical exercise besides physical education at school. There were a high proportion of inactive adolescents. Strategies to prevent physical inactivity in adolescents should be elaborated with a central role for the school and family.

Motor Activity; Adolescent; Maternal Behavior; Schools
Gabriel Gustavo Bergmann 1

Mauren Lúcia de Araújo Bergmann 1

Alexandre Carriconde Marques 2

Pedro Curi Hallal 2

\section{Resumo}

O objetivo do trabalho foi identificar a prevalência e os fatores associados à inatividade física em adolescentes. Estudo transversal com amostra de 1.455 adolescentes (741 do sexo feminino). Os dados foram coletados por meio de um questionário composto por indicadores sociodemográficos, de percepção e comportamentais. A atividade física foi estimada pelo Physical Activity Questionnaire for Older Children and Adolescents. A prevalência de inatividade física foi de 68\% (IC95\%: 65,6\%-70,4\%). Após análise ajustada permaneceram associadas $(p<0,05)$ à inatividade física: residir em apartamento, sexo feminino, idades mais avançadas, perceber-se menos ativo que os pares, percepção de nível de atividade física baixo da mãe, deslocamento passivo até a escola, não praticar educação física na escola, não gostar das aulas de educação física e baixo envolvimento em outras práticas corporais além da educação física escolar. A prevalência de adolescentes inativos foi elevada. Estratégias de combate à inatividade física em adolescentes devem ser elaboradas tendo a escola e a família papel de destaque.

Atividade Motora; Adolescente; Comportamento Materno; Instituições Acadêmicas 


\section{Introduction}

Physical activity is known to be a protective factor against various health problems. Physically active individuals show lower odds of developing cardiovascular and metabolic diseases, obesity, some types of cancer, osteoporosis, and psychological problems 1,2 . The population should thus be encouraged to maintain adequate levels of physical activity. However, population surveys have shown that this often fails to occur.

In Brazil, studies on physical activity have shown worrisome results. The proportion of physically inactive individuals is high. A systematic review identified 47 randomized studies on the prevalence of physical inactivity 3 . Of these, ten were conducted exclusively in children and adolescents. In adults, the prevalence rates for physical inactivity were similar to those found in the United States 4 and Europe 5,6, reaching more than $70 \%$ in some studies 7,8 . In the young population, prevalence rates for physical inactivity varied between studies, depending on the measurement procedures and classification, reaching greater than $90 \%$ in one study 9 .

Findings on physical inactivity in the young population in Brazil are already worrisome, due to the high prevalence. This concern is further aggravated by evidence from studies on the continuity of physical activity over life (or "tracking") and the secular trend. Tracking studies suggest reductions in levels of physical activity over life, especially in the transition from late childhood and adolescence to young adulthood 10,11. Recent decades have also witnessed an increase in physical inactivity even among children and adolescents 12,13 . This scenario suggests that physical inactivity will increase even more in future adult populations.

To improve our understanding of the problem and thus support the struggle against physical inactivity, we need to expand and extend our knowledge on physical activity in young populations. It is crucial to investigate the reasons leading young people to pursue an increasingly less physically active lifestyle, consisting mainly of activities that require little or no body movement. Some effort in this direction has already been made in Brazil 14,15,16,17,18. Still, few studies 16,17 have focused on the influence of factors such as physical education in school. Furthermore, we fail to find studies associating information on adolescents' self-rated physical activity or their perception of maternal physical activity.

Understanding different factors associated with physical activity can contribute to the development of effective programs to encourage physical exercise. This study thus aimed to identify the prevalence of physical inactivity and associated factors among adolescents 10 to 17 years of age.

\section{Methods}

This was a cross-sectional school-based study conducted with schoolchildren 10 to 17 years of age enrolled during the daytime in the municipal and state school systems in Uruguaiana, Rio Grande do Sul State, Brazil. Considering the sample calculation, 1,463 schoolchildren participated in the study. With this sample size, the margin of error for estimating the proportion was 2.8 percentage points. In order for the number of adolescents from the rural area to represent the proportion of rural students enrolled, it would be necessary to include 95 adolescents.

The study adopted probabilistic cluster sampling, where each school was considered a cluster. All the public schools in the municipality participated in the selection round and had the same odds of being selected to participate in the study according to the number of enrolled students in the 10 to 17 year age bracket. To reach the estimated number of adolescents, it was necessary to pick 10 schools (nine urban, seven of which state and two municipal, plus one rural). All the schoolchildren 10 to 17 years of age from the 10 selected schools were invited to participate. The final sample only included those who turned in the informed consent form signed by a parent or guardian and who expressed their own desire to participate.

Data collection involved applying a questionnaire organized in sections to all the individuals comprising the sample. The questionnaire contained questions on: (a) demographics; (b) routine daily behaviors and habits; (c) self-rated physical activity and nutritional status and perception of maternal physical activity; and (d) leisure-time physical activity and habits.

Physical activity (dependent variable) was estimated using the Physical Activity Questionnaire for Older Children - PAQ-C 19 and Adolescents - PAQ-A 20, adapted and validated for Brazil as reported by Silva \& Malina 21 . The instrument consists of nine questions on sports, games, and other physical activities in school and in leisure time in the previous seven days, including the weekend. Each question has five possible answers (values from 1 to 5), allowing the creation of a score. The score divided by the number of questions allows creating five categories of physical activity, classifying individuals as very sedentary, sedentary, moderately active, active, or very active. However, in our study we chose to replace the expressions "very sedentary" and "sedentary" 
with "very inactive" and "inactive", respectively. In agreement with other authors 22 , we contend that even when individuals display sedentary behaviors or a sedentary lifestyle they can be considered active, given that "sedentarism" is defined as the time spent per day in the sitting position and not as the lack of physical activity.

The independent variables were organized in three blocks: socio-demographic indicators, perceptual indicators, and behavioral indicators. Socio-demographic indicators were defined as: (a) socioeconomic status (according to the Brazilian Economic Classification Criterion 23 - divided into five levels from "A" to "E"); (b) headof-family's schooling ( $\geq 13$ years, 9-12 years, 8-5 years, and $<5$ years); (c) area of residence (urban versus rural); d) type of residence (house versus apartment); (d) school system (state versus municipal); (e) gender (male or female); and (f) age (in complete years).

The perceptual indicators were: (a) self-rated physical activity (much less active than others; less active than others; as active as others; more active than others; or much more active than others); (b) perception of maternal nutritional status (normal weight; overweight; or obese); and (c) perception of level of maternal physical activity (active versus inactive).

The behavioral indicators were: (a) commuting to school (active versus passive); (b) attends physical education in school (yes or no); (c) enjoys physical education in school (yes or no); (d) enjoys physical exercise (yes or no); (e) screen time, or time at TV, videogames, and computer ( $\leq 3$ hours versus $>3$ hours per day); (f) smoking (never smoked; former smoker; or regular smoker); and (g) alcohol consumption (never drank; used to drink; or drinks regularly).

Although commuting to school is part of physical activity for the adolescents (different domains of physical activity), the PAQ-C and PAQ-A provide a generalized classification of physical activity. We thus chose to use commuting to school as an independent variable in order to test whether this behavior was associated with the classification of physical activity in the instrument we used.

Data quality control used dual keying-in. The data were stored in a databank formatted in Epidata (Epidata Assoc., Odense, Denmark). After verifying the data's consistency, the databank was exported to SPSS for Windows 17.0 (SPSS Inc., Chicago, USA) where it was analyzed. Univariate, bivariate, and multivariate analyses were performed.

The univariate analysis used absolute frequencies and relative frequencies (proportions) in each of the study variables, followed by cal- culation of the $95 \%$ confidence interval (95\%CI). Bivariate analysis used chi-square test for heterogeneity and chi-square for linear trend. In this analysis, each independent variable was associated with the dichotomized dependent variable (the categories very inactive and inactive were grouped as "inactive", and the categories moderately active, active, and very active were grouped as "active").

The multivariate analysis used binary logistic regression with dichotomized physical activity as the outcome. The entry of each independent variable in this analysis followed the constructed hierarchical theoretical model (Figure 1). As factors associated with physical activity (inactive), the final multivariate model included independent variables with $\mathrm{p}<0.05$.

The study was approved by the respective institutional review board (case n. 042/2010). Data were collected from April to August 2011 by previously trained physical education students and faculty.

\section{Results}

A total of 1,463 adolescents were evaluated. Of these, eight were finally excluded because they fell outside the target age bracket. Of the 1,455 adolescents with consistent data, 1,343 completed all the information for calculating the score on level of physical activity. Table 1 shows the descriptive results of the study variables. The results call attention to the high prevalence of adolescents classified as very inactive or inactive $(68 \%)$.

Due to the low frequency in given categories of the independent variables socioeconomic status, smoking, and perception of maternal nutritional status, we chose to group them with other categories when performing the bivariate and multivariate analyses.

In the bivariate analysis, of the 17 independent study variables compared to the dependent variable, only five failed to show a statistically significant association with physical activity $(\mathrm{p} \geq$ 0.05) (Table 2).

The crude odds ratios (OR) in the binary logistic regression confirmed the results of the chi-square test. When analyzed jointly (adjusted $\mathrm{OR})$, some variables lost statistical significance and did not enter the final multivariate model. The results of the adjusted binary logistic regression showed that living in an apartment, female gender, older adolescence, self-perception as less active than one's peers, perception of one's mother as inactive, not walking or bicycling to school, not participating in physical education in school, and not enjoying physical education 


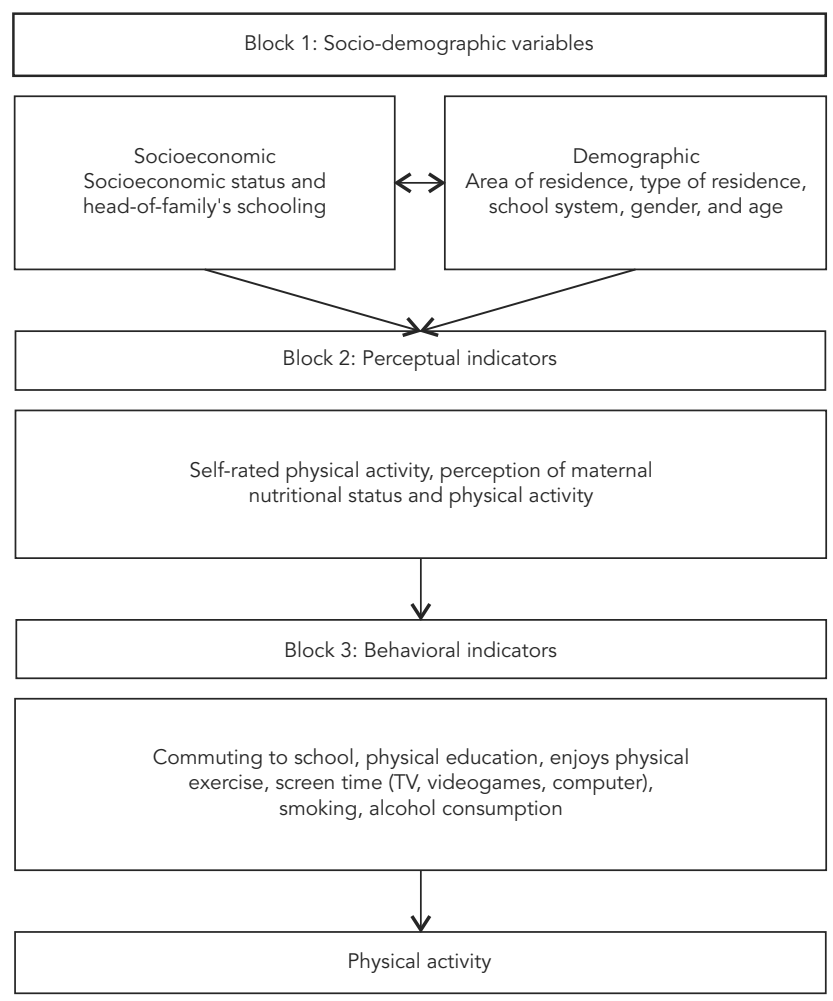

classes in school increased the odds $(\mathrm{p}<0.05)$ of adolescents 10 to 17 years of age being classified as inactive (Table 3 ), forming the final theoretical model between physical activity (inactive) and associated factors in adolescents.

\section{Discussion}

Our study's results indicated that more than two-thirds of the sample were classified as physically inactive. This proportion varied according to stratification of the sample in subgroups (independent study variables). This procedure, in addition to identifying the factors associated with physical inactivity in adolescents 10 to 17 years of age, can contribute to the elaboration of programs to prevent physical inactivity and to promote adherence to regular physical exercise.

Brazilian studies with representative samples using the same instrument showed similar prevalence rates 14,24 or higher ones 21,25 than the rate of inactive or very inactive individuals found here. A comparison of our findings with those of studies with representative samples of adolescents that used as the classification procedure the current recommendations for physical activity for adolescents $(\geq 300$ minutes per week of physical activities) showed varied results. In a study of 1,518 adolescents 14 to 18 years of age in Curitiba, Paraná State, Brazil 26, the authors found that $85.5 \%$ of the individuals failed to meet the recommended amount of physical activity. Using the same classification criterion, Hallal et al. 16, studying a representative sample of adolescents 10 to 12 years of age in Pelotas, Rio Grande do Sul State, Brazil, found $58.2 \%$ of inactive individuals. In a study of 4,210 adolescents (14-19 years) in Pernambuco State 27, the authors found $65.1 \%$ of physically inactive individuals. A sample of more than 60,000 ninth graders from all the Brazilian state capitals and the Federal District under the National School Health Survey (PeNSE) found $56.9 \%$ of physical inactivity, with no major differences in prevalence between regions of the country 28 . 
Table 1

Findings on variables studied in adolescents 10 to 17 years of age in Uruguaiana, Rio Grande do Sul State, Brazil, 2011.

\begin{tabular}{|c|c|c|}
\hline Variables & $\mathrm{n}$ & $\%(95 \% \mathrm{Cl})$ \\
\hline \multicolumn{3}{|l|}{ Dependent } \\
\hline \multicolumn{3}{|l|}{ Physical activity } \\
\hline Very inactive & 360 & $26.8(24.4-29.2)$ \\
\hline Inactive & 553 & $41.2(38.6-43.8)$ \\
\hline Moderately active & 318 & $23.7(21.4-26.0)$ \\
\hline Active & 102 & $7.6(6.2-9.0)$ \\
\hline Very active & 10 & $0.7(0.2-1.1)$ \\
\hline \multicolumn{3}{|l|}{ Socio-demographic } \\
\hline \multicolumn{3}{|l|}{ Socioeconomic status } \\
\hline B & 263 & $20.6(14.3-18.3)$ \\
\hline C & 817 & $63.9(61.3-66.5)$ \\
\hline $\mathrm{D}$ & 190 & $14.9(13.0-16.8)$ \\
\hline$E$ & 8 & $0.6(0.2-1.0)$ \\
\hline \multicolumn{3}{|l|}{ Head-of-family's schooling (years) } \\
\hline$\geq 13$ & 223 & $16.3(14.3-18.3)$ \\
\hline 9-12 & 498 & $36.3(33.8-38.8)$ \\
\hline $5-8$ & 327 & $23.9(21.6-26.2)$ \\
\hline$\leq 4$ & 323 & $23.6(21.3-25.8)$ \\
\hline \multicolumn{3}{|l|}{ Area of residence } \\
\hline Urban & 1,296 & $90.8(89.3-92.3)$ \\
\hline Rural & 131 & $9.2(7.7-10.7)$ \\
\hline \multicolumn{3}{|l|}{ Type of residence } \\
\hline House & 1,345 & $94.1(92.9-95.3)$ \\
\hline Apartment & 84 & $5.9(4.7-7.1)$ \\
\hline \multicolumn{3}{|l|}{ School system } \\
\hline State & 993 & $68.2(65.8-70.6)$ \\
\hline Municipal & 462 & $31.8(29.4-34.2)$ \\
\hline \multicolumn{3}{|l|}{ Gender } \\
\hline Male & 714 & $49.1(46.5-51.7)$ \\
\hline Female & 741 & $50.9(48.3-53.5)$ \\
\hline \multicolumn{3}{|l|}{ Age (years) } \\
\hline 10 & 79 & $5.4(4.2-6.6)$ \\
\hline 11 & 209 & $14.4(12.6-16.2)$ \\
\hline 12 & 205 & $14.1(12.3-15.9)$ \\
\hline 13 & 232 & $15.9(14.0-17.8)$ \\
\hline 14 & 211 & $14.5(12.7-16.3)$ \\
\hline 15 & 221 & $15.2(13.3-17.1)$ \\
\hline 16 & 174 & $12.0(10.3-13.7)$ \\
\hline 17 & 124 & $8.5(7.1-9.9)$ \\
\hline \multicolumn{3}{|l|}{ Perception of physical activity } \\
\hline \multicolumn{3}{|l|}{ Self-rated physical activity } \\
\hline Much less active than others & 201 & $14.0(12.2-15.8)$ \\
\hline Less active than others & 286 & $20.0(17.9-22.1)$ \\
\hline As active as others & 504 & $35.2(32.7-37.7)$ \\
\hline More active than others & 301 & $21.0(18.9-23.1)$ \\
\hline Much more active than others & 139 & $9.7(8.2-11.2)$ \\
\hline \multicolumn{3}{|c|}{ Perception of maternal nutritional status } \\
\hline Normal weight & 684 & $48.0(45.4-50.6)$ \\
\hline Overweight & 703 & $49.3(46.7-51.9)$ \\
\hline Obese & 39 & $2.7(1.9-3.5)$ \\
\hline
\end{tabular}

(continues) 


\begin{tabular}{|c|c|c|}
\hline Variables & $\mathrm{n}$ & $\%(95 \% \mathrm{Cl})$ \\
\hline \multicolumn{3}{|c|}{ Perception of maternal physical activity } \\
\hline Inactive & 712 & $50.1(47.5-52.7)$ \\
\hline Active & 710 & $49.9(47.3-52.5)$ \\
\hline \multicolumn{3}{|l|}{ Behavioral indicators } \\
\hline \multicolumn{3}{|l|}{ Commuting to school } \\
\hline Active & 1,082 & 75.7 (73.5-77.9) \\
\hline Passive & 348 & $24.3(22.1-26.5)$ \\
\hline \multicolumn{3}{|l|}{ Physical education in school } \\
\hline Yes & 1,191 & $84.6(82.7-86.5)$ \\
\hline No & 216 & $15.4(13.5-17.3)$ \\
\hline \multicolumn{3}{|l|}{ Enjoys physical education classes } \\
\hline Yes & 1,009 & $79.2(77.0-81.4)$ \\
\hline No & 265 & $20.8(18.6-23.0)$ \\
\hline \multicolumn{3}{|l|}{ Enjoys physical exercise } \\
\hline Yes & 1,251 & $87.5(85.8-89.2)$ \\
\hline No & 178 & $12.5(10.8-14.2)$ \\
\hline \multicolumn{3}{|c|}{$\begin{array}{l}\text { Screen time (TV, videogames, computer - } \\
\text { hours/day) }\end{array}$} \\
\hline$\leq 3$ & 521 & $35.8(33.3-38.3)$ \\
\hline$>3$ & 934 & $64.2(61.7-66.7)$ \\
\hline \multicolumn{3}{|l|}{ Smoking } \\
\hline Never smoked & 1,283 & $89.6(88.0-91.2)$ \\
\hline Former smoker & 123 & $8.6(7.2-10.0)$ \\
\hline Regular smoker & 26 & $1.8(1.1-2.5)$ \\
\hline \multicolumn{3}{|l|}{ Alcohol consumption } \\
\hline Never drank & 803 & $56.1(53.5-58.7)$ \\
\hline Used to drink, no longer drinks & 336 & $23.5(21.3-25.7)$ \\
\hline Drinks regularly & 293 & $20.5(18.4-22.6)$ \\
\hline
\end{tabular}

95\%Cl: $95 \%$ confidence interval.

Table 2

Bivariate analysis between level of physical activity (inactive or active) and independent study variables among adolescents in Uruguaiana, Rio Grande do Sul State, Brazil, 2011.

\begin{tabular}{|c|c|c|c|c|}
\hline \multirow[t]{3}{*}{ Variables } & \multirow[t]{3}{*}{ n (\%) } & \multicolumn{2}{|c|}{ Physical activity } & \multirow[t]{3}{*}{$p$-value } \\
\hline & & Inactive & Active & \\
\hline & & $\%(95 \% \mathrm{Cl})$ & $\%(95 \% \mathrm{Cl})$ & \\
\hline \multicolumn{5}{|l|}{ Socio-demographic } \\
\hline Socioeconomic status * & & & & 0.562 \\
\hline$B$ & $248(20.8)$ & $68.1(62.3-73.9)$ & $31.9(26.1-37.7)$ & \\
\hline C & $796(64.5)$ & $67.0(63.7-70.3)$ & $33.0(29.7-36.3)$ & \\
\hline$D / E$ & $175(14.7)$ & $71.4(64.7-78.1)$ & $28.6(21.9-35.3)$ & \\
\hline Head-of-family's schooling (years) * & & & & 0.780 \\
\hline$\geq 13$ & 204 (15.9) & $69.6(63.3-75.9)$ & $30.4(24.1-36.7)$ & \\
\hline $9-12$ & $459(35.8)$ & $69.7(65.5-73.9)$ & $30.3(26.1-34.5)$ & \\
\hline $5-8$ & $303(24.6)$ & $61.7(56.2-67.2)$ & $38.3(32.8-43.8)$ & \\
\hline$\leq 4$ & $316(24.6)$ & $70.9(65.9-75.9)$ & $29.1(24.1-34.1)$ & \\
\hline
\end{tabular}

(continues) 


\begin{tabular}{|c|c|c|c|c|}
\hline \multirow[t]{3}{*}{ Variables } & \multirow[t]{3}{*}{$\mathrm{n}(\%)$} & \multicolumn{2}{|c|}{ Physical activity } & \multirow[t]{3}{*}{ p-value } \\
\hline & & Inactive & Active & \\
\hline & & $\%(95 \% \mathrm{Cl})$ & $\%(95 \% \mathrm{Cl})$ & \\
\hline \multicolumn{5}{|l|}{ Socio-demographic } \\
\hline Area of residence ** & & & & 0.021 \\
\hline Urban & $1,238(92.7)$ & $69.1(66.5-71.7)$ & $30.9(28.3-33.5)$ & \\
\hline Rural & $97(7.3)$ & $57.7(47.9-67.5)$ & $42.3(32.5-52.1)$ & \\
\hline Type of residence ** & & & & 0.011 \\
\hline House & $1,263(94.4)$ & $67.3(64.7-69.9)$ & $32.7(30.1-35.3)$ & \\
\hline Apartment & $75(5.6)$ & $81.3(72.5-90.1)$ & $18.7(19.5-40.3)$ & \\
\hline School system ** & & & & 0.015 \\
\hline Municipal & $421(31.3)$ & $63.4(58.8-68.0)$ & $36.6(32.0-41.2)$ & \\
\hline State & $922(68.7)$ & $70.1(67.1-73.1)$ & $29.9(27.0-32.8)$ & \\
\hline Gender ** & & & & 0.000 \\
\hline Male & $663(49.4)$ & $53.1(49.3-56.9)$ & $46.9(43.1-50.7)$ & \\
\hline Female & $680(50.6)$ & $82.5(79.6-85.4)$ & $17.5(26.5-33.3)$ & \\
\hline Age (years) * & & & & 0.000 \\
\hline 10 & $74(5.5)$ & $51.4(40.0-62.8)$ & $48.6(37.2-60.0)$ & \\
\hline 11 & $200(14.9)$ & $57.5(50.7-64.3)$ & $42.5(35.7-49.3)$ & \\
\hline 12 & $195(14.5)$ & $64.1(57.4-70.8)$ & $35.9(29.2-42.6)$ & \\
\hline 13 & $217(16.2)$ & $62.2(55.8-68.6)$ & $37.8(31.4-44.4)$ & \\
\hline 14 & $199(16.2)$ & $68.3(61.8-74.8)$ & $31.7(25.2-38.2)$ & \\
\hline 15 & $203(15.1)$ & $79.8(74.3-85.3)$ & $20.2(14.7-25.7)$ & \\
\hline 16 & $151(11.2)$ & $78.1(71.5-84.7)$ & $21.9(15.3-28.5)$ & \\
\hline 17 & $104(7.7)$ & $80.8(73.2-88.4)$ & $19.2(11.6-26.8)$ & \\
\hline \multicolumn{5}{|l|}{ Perceptual indicators } \\
\hline Self-rated physical activity * & & & & 0.000 \\
\hline Much less active than others & $192(14.3)$ & $87.5(82.8-92.2)$ & $12.5(7.8-17.2)$ & \\
\hline Less active than others & $274(20.4)$ & $85.8(81.7-89.9)$ & $14.2(10.1-18.3)$ & \\
\hline As active as others & $465(34.6)$ & $66.7(62.4-71.0)$ & $33.3(29.0-37.6)$ & \\
\hline More active than others & $282(21.0)$ & $55.3(49.5-61.1)$ & $44.7(38.9-50.5)$ & \\
\hline Much more active than others & $129(9.6)$ & $34.1(25.9-42.3)$ & $65.9(57.7-74.1)$ & \\
\hline $\begin{array}{l}\text { Perception of maternal nutritional } \\
\text { status ** }\end{array}$ & & & & 0.298 \\
\hline Normal weight & $646(48.4)$ & $66.7(36.1-70.3)$ & $33.3(29.7-36.9)$ & \\
\hline Overweight/Obese & $689(48.8)$ & $69.4(63.2-70.2)$ & $30.6(27.2-34.0)$ & \\
\hline $\begin{array}{l}\text { Perception of maternal physical } \\
\text { activity *夫 }\end{array}$ & & & & 0.001 \\
\hline Inactive & $677(50.9)$ & $72.5(69.1-75.9)$ & $27.5(24.1-30.9)$ & \\
\hline Active & $654(49.1)$ & $63.8(60.1-67.5)$ & $36.2(32.5-39.9)$ & \\
\hline \multicolumn{5}{|l|}{ Behavioral indicators } \\
\hline Commuting to school ** & & & & 0.068 \\
\hline Active & $1,045(78.2)$ & $67.0(64.2-69.8)$ & $33.0(30.2-35.8)$ & \\
\hline Passive & $292(21.8)$ & $72.6(67.5-77.7)$ & $27.4(22.3-32.5)$ & \\
\hline Physical education in school ** & & & & 0.000 \\
\hline Yes & $1,118(84.7)$ & $63.7(60.9-66.5)$ & $36.3(33.5-39.1)$ & \\
\hline No & $202(15.3)$ & $91.1(87.2-95.0)$ & $8.9(21.3-33.5)$ & \\
\hline Enjoys physical education classes ** & & & & 0.000 \\
\hline Yes & $947(79.3)$ & $62.0(58.9-65.1)$ & $38.0(34.9-41.1)$ & \\
\hline No & $247(20.7)$ & $88.3(84.3-92.3)$ & $11.7(7.7-15.7)$ & \\
\hline
\end{tabular}

(continues) 


\begin{tabular}{|c|c|c|c|c|}
\hline \multirow[t]{3}{*}{ Variables } & \multirow[t]{3}{*}{$n(\%)$} & \multicolumn{2}{|c|}{ Physical activity } & \multirow[t]{3}{*}{$\mathrm{p}$-value } \\
\hline & & Inactive & Active & \\
\hline & & $\%(95 \% \mathrm{Cl})$ & $\%(95 \% \mathrm{Cl})$ & \\
\hline \multicolumn{5}{|l|}{ Behavioral indicators } \\
\hline Enjoys physical exercise ** & & & & 0.001 \\
\hline Yes & $1,170(87.4)$ & $66.5(63.8-69.2)$ & $33.5(30.8-36.2)$ & \\
\hline No & $168(12.6)$ & $79.8(73.7-85.9)$ & $20.2(14.1-26.3)$ & \\
\hline $\begin{array}{l}\text { Screen time (TV, videogames, } \\
\text { computer - hours/day) }{ }^{* *}\end{array}$ & & & & 0.003 \\
\hline$\leq 3$ & $484(36.0)$ & $63.0(58.7-67.3)$ & $37.0(32.7-41.3)$ & \\
\hline$>3$ & $859(64.0)$ & $70.8(67.8-73.8)$ & $29.2(17.5-22-9)$ & \\
\hline Smoking ** & & & & 0.126 \\
\hline Never smoked & $1,204(89.8)$ & $67.3(64.6-70.0)$ & $32.7(30.0-35.4)$ & \\
\hline Former smoker/Regular smoker & $137(10.2)$ & $73.7(66.3-81.1)$ & $26.3(18.9-33.7)$ & \\
\hline Alcohol consumption * & & & & 0.000 \\
\hline Never drank & $768(57.3)$ & $65.4(62.0-68.8)$ & $34.6(31.2-38.0)$ & \\
\hline Used to drink, no longer drinks & $313(23.3)$ & $69.3(64.2-74.4)$ & $30.7(25.6-35.8)$ & \\
\hline Drinks regularly & $260(19.4)$ & $74.2(68.9-79.5)$ & $25.8(20.5-31.1)$ & \\
\hline
\end{tabular}

95\% Cl: $95 \%$ confidence interval.

${ }^{*}$ Chi-square test for trend;

** Chi-square test for heterogeneity.

Table 3

Crude and adjusted odds ratio for physical activity (inactive versus active) and associated factors among adolescents in Uruguaiana, Rio Grande do Sul State, Brazil, 2011.

\begin{tabular}{|c|c|c|c|c|c|}
\hline \multirow[t]{2}{*}{ Variables } & \multirow[t]{2}{*}{ n (\%) } & \multicolumn{4}{|c|}{ Bivariate logistic regression } \\
\hline & & Crude OR $(95 \% \mathrm{Cl})$ & $\mathrm{p}$-value & Adjusted OR $(95 \% \mathrm{Cl})$ & p-value \\
\hline \multicolumn{6}{|c|}{ Socio-demographic } \\
\hline \multicolumn{6}{|c|}{ Socioeconomic status } \\
\hline B & $248(20.8)$ & 1.00 & - & 1.00 & - \\
\hline C & $796(64.5)$ & $0.95(0.70-1.29)$ & 0.732 & $0.92(0.60-1.40)$ & 0.683 \\
\hline $\mathrm{D} / \mathrm{E}$ & $175(14.7)$ & $1.17(0.77-1.79)$ & 0.470 & $1.14(0.64-2.04)$ & 0.657 \\
\hline \multicolumn{6}{|c|}{ Head-of-family's schooling (years) } \\
\hline$\geq 13$ & 204 (15.9) & 1.00 & - & 1.00 & - \\
\hline $9-12$ & $459(35.8)$ & $1.00(0.70-1.44)$ & 0.978 & $0.99(0.62-1.58)$ & 0.959 \\
\hline $5-8$ & $303(24.6)$ & $0.70(0.48-1.02)$ & 0.068 & $0.77(0.46-1.29)$ & 0.327 \\
\hline$\leq 4$ & $316(24.6)$ & $1.06(0.72-1.56)$ & 0.755 & $0.89(0.51-1.53)$ & 0.670 \\
\hline \multicolumn{6}{|c|}{ Area of residence } \\
\hline Urban & $1,238(92.7)$ & $1.63(1.07-2.49)$ & 0.022 & $1.57(0.93-2.65)$ & 0.090 \\
\hline Rural & $97(7.3)$ & 1.00 & - & 1.00 & - \\
\hline \multicolumn{6}{|c|}{ Type of residence } \\
\hline House & $1,263(94.4)$ & 1.00 & - & 1.00 & - \\
\hline Apartment & $75(5.6)$ & $2.12(1.17-3.83)$ & 0.013 & $1.90(1.00-3.60)$ & 0.048 \\
\hline \multicolumn{6}{|l|}{ School system } \\
\hline Municipal & $421(31.3)$ & 1.00 & - & 1.00 & - \\
\hline State & $922(68.7)$ & $1.35(1.06-1.72)$ & 0.016 & $1.15(0.85-1.56)$ & 0.355 \\
\hline
\end{tabular}

(continues) 


\begin{tabular}{|c|c|c|c|c|c|}
\hline \multirow[t]{2}{*}{ Variables } & \multirow[t]{2}{*}{ n (\%) } & \multicolumn{4}{|c|}{ Bivariate logistic regression } \\
\hline & & Crude OR $(95 \% \mathrm{Cl})$ & $\mathrm{p}$-value & Adjusted OR $(95 \% \mathrm{Cl})$ & $\mathrm{p}$-value \\
\hline \multicolumn{6}{|l|}{ Socio-demographic } \\
\hline \multicolumn{6}{|l|}{ Gender } \\
\hline Male & $663(49.4)$ & 1.00 & - & 1.00 & - \\
\hline Female & $680(50.6)$ & $4.16(3.24-5.35)$ & 0.000 & $4.33(3.29-5.69)$ & 0.000 \\
\hline \multicolumn{6}{|l|}{ Age (years) } \\
\hline $10-17$ & $1,343(100.0)$ & $1.23(1.16-1.31)$ & 0.000 & $1.24(1.155-1.332)$ & 0.000 \\
\hline \multicolumn{6}{|l|}{ Perceptual indicators } \\
\hline \multicolumn{6}{|l|}{ Self-rated physical activity } \\
\hline Much less active than others & $192(14.3)$ & $13.52(7.71-23.71)$ & 0.000 & $16.70(8.36-33.32)$ & 0.000 \\
\hline Less active than others & $274(20.4)$ & $11.64(7.08-19.14)$ & 0.000 & $9.66(5.42-17.22)$ & 0.000 \\
\hline As active as others & $465(34.6)$ & $3.86(2.56-5.83)$ & 0.000 & $4.37(2.66-7.20)$ & 0.000 \\
\hline More active than others & $282(21.0)$ & $2.39(1.55-3.69)$ & 0.000 & $2.59(1.55-4.35)$ & 0.000 \\
\hline Much more active than others & $129(9.6)$ & 1.00 & - & 1.00 & - \\
\hline \multicolumn{6}{|l|}{$\begin{array}{l}\text { Perception of maternal nutritional } \\
\text { status }\end{array}$} \\
\hline Normal weight & $646(48.4)$ & 1.00 & - & 1.00 & - \\
\hline Overweight/Obese & $689(48.8)$ & $1.13(0.90-1.42)$ & 0.298 & $0.98(0.743-1.31)$ & 0.897 \\
\hline \multicolumn{6}{|l|}{$\begin{array}{l}\text { Perception of maternal physical } \\
\text { activity }\end{array}$} \\
\hline Inactive & $677(50.9)$ & $1.50(1.19-189)$ & 0.001 & $1.36(1.02-1.82)$ & 0.037 \\
\hline Active & $654(49.1)$ & 1.00 & - & 1.00 & - \\
\hline \multicolumn{6}{|l|}{ Behavioral indicators } \\
\hline \multicolumn{6}{|l|}{ Commuting to school } \\
\hline Active & $1,045(78.2)$ & 1.00 & - & 1.00 & - \\
\hline Passive & $292(21.8)$ & $1.31(0.98-1.74)$ & 0.069 & $1.62(1.04-2.54)$ & 0.035 \\
\hline \multicolumn{6}{|l|}{ Physical education in school } \\
\hline Yes & $1,118(84.7)$ & 1.00 & - & 1.00 & - \\
\hline No & $202(15.3)$ & $5.83(3.54-9.60)$ & 0.000 & $2.83(1.14-6.99)$ & 0.024 \\
\hline \multicolumn{6}{|l|}{ Enjoys physical education classes } \\
\hline Yes & $947(79.3)$ & 1.00 & - & 1.00 & - \\
\hline No & $247(20.7)$ & $4.61(3.06-6.94)$ & 0.000 & $2.02(1.05-3.89)$ & 0.036 \\
\hline \multicolumn{6}{|l|}{ Enjoys physical exercise } \\
\hline Yes & $1,170(87.4)$ & 1.00 & - & 1.00 & - \\
\hline No & $168(12.6)$ & $1.99(1.34-2.95)$ & 0.001 & $1.77(0.99-3.17)$ & 0.054 \\
\hline \multicolumn{6}{|l|}{$\begin{array}{l}\text { Screen time (TV, videogames, } \\
\text { computer - hours/day) }\end{array}$} \\
\hline$\leq 3$ & $484(36.0)$ & 1.00 & - & 1.00 & - \\
\hline$>3$ & $859(64.0)$ & $1.42(1.12-1.80)$ & 0.003 & $1.33(0.96-1.82)$ & 0.083 \\
\hline \multicolumn{6}{|l|}{ Smoking } \\
\hline Never smoked & 1,204 (89.8) & 1.00 & - & 1.00 & - \\
\hline Formers smoker/Regular smoker & $137(10.2)$ & $1.36(0.92-2.04)$ & 0.127 & $0.77(0.43-1.41)$ & 0.409 \\
\hline \multicolumn{6}{|l|}{ Alcohol consumption } \\
\hline Never drank & $768(57.3)$ & 1.00 & - & 1.00 & - \\
\hline Used to drink, no longer drinks & $313(23.3)$ & $1.20(0.90-1.59)$ & 0.211 & $1.03(0.70-1.53)$ & 0.868 \\
\hline Drinks regularly & $260(19.4)$ & $1.53(1.11-2.09)$ & 0.009 & $0.85(0.52-1.40)$ & 0.534 \\
\hline
\end{tabular}

95\% Cl: 95\% confidence interval; OR: odds ratio; p-value: level of significance. 
Although diverse measurement instruments and classification procedures are used in studies on physical activity in children and adolescents and add to the disparate seasonal, environmental, and cultural characteristics to hinder comparison between studies, the low prevalence of active individuals in different studies is recurrent, regardless of the procedure. Among the above-mentioned studies, none showed more than $45 \%$ of active individuals. These results are similar to those presented in a study 29 that combined information from 35 countries of Europe and North America, identifying a prevalence rate of only $35.3 \%$ of young individuals classified as active, and a study 30 of adolescents from 100 American cities with $48 \%$ of active individuals. These results indicate that physical inactivity can be considered a worldwide phenomenon and emphasizes the need for studies on the determinants of physical activity in children and adolescents in order to provide information for the elaboration of programs to encourage physical exercise.

In relation to associated factors, our results indicated that adolescents living in apartments showed higher odds of being inactive when compared to those living in houses. This finding may be related to the more ample spaces available for physical exercise in houses, which in many cases have yards or patios. According to a study by Babey et al. 31 including 4,010 adolescents 12-17 years of age in California, USA, physical inactivity was more common in adolescents that lived in houses. However, Hallal et al. 16 did not find an association between physical activity and type of residence in their study of adolescents in Pelotas. More studies are needed on physical activity in children and adolescents including type of residence as an independent variable in order to understand its possible impact.

In relation to gender and age, our results are consistent with the literature. Female gender and older age brackets showed higher rates of inactivity. Studies in different regions of Brazil $18,21,25,26$ and in other countries 13,32,33,34 agree with these findings. Importantly, however, there are studies indicating no association between these two variables and physical activity in adolescents 17,35 .

In programs to promote physical activity, among other characteristics, it is essential for individuals not only to understand the benefits of a physically active life but also to perceive themselves as active or inactive; in the former case, they should remain active, and in the latter they should modify their lifestyle. The results of the current study indicate that inactive adolescents are aware of their situation. The association with self-rated physical activity had received little attention in previous studies and can facilitate understanding of the reasons leading adolescents to adopt such lifestyle and thus elaborate strategies to change this situation.

Various studies have analyzed family influence on levels of physical activity in children and adolescents. Among the possible family factors, level of maternal physical activity appears as an independent variable in various studies of physical activity in adolescents 16,36,37. The results indicate that adolescents with inactive mothers show higher odds of being inactive themselves, although other studies fail to confirm these associations with the mother, only with the father 38 , while others fail to confirm associations with either parent 39 .

Although part of the available evidence indicates that parents' physical activity is associated with physical activity in the children, this possible influence may be understood better by analyzing the children's perception of the level of their parents' physical activity. Our findings suggest that adolescents who saw their mother as inactive showed higher odds of being inactive themselves (OR $=1.49$; 95\%CI: 1.10-2.02). Some recent international studies have also included this analysis 40,41 . In a sample of 1,004 students nine to 18 years of age in Mexico, Siegel et al. 40 identified perception of maternal participation in sports and physical activities as a significant predictor of variance in the PAQ score in adolescents. In a study of 1,978 adolescents 12 to 19 years of age in La Rioja, Spain, the authors found that adolescents who saw their mothers as inactive showed 3.5 times higher odds of being inactive when compared to their peers that saw their mothers as active 41 .

In our study, adolescents that reported commuting to school passively showed higher odds of being classified as inactive. Although this variable belong to one of the domains of physical activity (commuting), since our instrument did not include this type of information when calculating the final physical activity score, it was inserted as an independent variable in our analyses. Other studies 16,42 analyzed commuting to school as a dependent variable, indicating this behavior's importance for physical activity in adolescents. However, our results are important if we consider the high prevalence rates of individuals classified as inactive (68\%) and who report not having any type of exercise except for physical education in school (74.9\%), indicating that for a large portion of these adolescents, commuting to school become the only regular physical activity besides physical education in school. 
Our study's findings demonstrate the importance of physical education in school for levels of physical activity in adolescents. Individuals that report not participating in physical education classes $(\mathrm{OR}=4.02$; 95\%CI: $1.62-9.99)$ or that report not liking the classes $(\mathrm{OR}=2.06$; $95 \% \mathrm{CI}$ : 1.06-4.02) show significantly higher odds of being inactive. Other studies corroborate our results, indicating that participation in physical education classes in school increases the odds of adolescents being active 16,17,43 and that the odds increase further when participation occurs five times a week $17,43,44$.

When we included the variables commuting to school and physical education in school as independent variables, we were aware of both the positive aspects and the limitations of this methodological choice. The positive aspects include the result indicating that the PAQ-C and PAQ-A classification (which can be considered a general measure of physical activity) is consistent with other indicators of physical activity. However, this methodological choice means combining different behaviors of physical activity in a general classification. Thus, rather than considering these variables as factors associated with physical activity in adolescents, it might be more adequate to view them as integral parts of such activity.

The current study presents some limitations for generalization since it deals with a sample of children from the public school system, thus impeding inference due to the lack of individuals with higher socioeconomic status, who are present in both the private school system and the vocational training schools. In addition, the target age bracket is in a moment of change vis-à-vis possibilities for participation in the labor market, but this variable was not measured to verify the potential association with leisure-time physical activity. Although the study showed significant associations between various independent variables and physical activity, it was not possible to establish causality since this was a cross-sectional study. In addition, information on physical activity is subject to recall bias since it refers to activities performed in the previous seven days. Still, regardless of the above-mentioned limitations, the study's findings are representative and contribute to the body of knowledge on physical activity and associated factors in adolescents 10 to 17 years of age.

\section{Conclusions}

The study confirmed the high proportion of inactive children and adolescents. The identification of an association between inactivity and non-participation in physical education at school and lack of enjoyment of such classes, plus the perception of one's own reduced physical activity and that of one's mother assign significant importance to the school and family in the fight against physical inactivity in children and adolescents.

\section{Resumen}

Identificar la prevalencia y los factores asociados a la inactividad física en adolescentes. Estudio transversal con una muestra de 1.455 adolescentes (741 de sexo femenino). Los datos fueron recogidos mediante un cuestionario compuesto por indicadores sociodemográficos, de percepción y de comportamiento. La actividad física fue estimada por el Physical Activity Questionnaire for Older Children and Adolescents. La prevalencia de inactividad física fue de un 68\% (IC95\%: 65,6\%-70,4\%). Tras el análisis ajustado permanecieron asociadas (valor de $p<0,05$ ) a la inactividad física: residir en un apartamento, sexo femenino, edades más avanzadas, percibirse menos activo que la pareja, percepción de ni- vel de actividad física bajo de la madre, desplazamiento pasivo hasta la escuela, no practica educación física en la escuela, no le gustan las clases de educación física, y tienen poca relación con otras prácticas corporales, además de la educación física escolar. La prevalencia de adolescentes inactivos fue elevada. Se deben elaborar estrategias de combate a la inactividad física en adolescentes, considerando el papel relevante de la escuela y la familia.

Actividad Motora; Adolescente; Conducta Materna; Instituciones Académicas 


\section{Contributors}

G. G. Bergmann and M. L. A. Bergmann participated in all stages of the study's production and approved the final version for publication. A. C. Marques and P. C. Hallal participated in the data analysis and interpretation and relevant critical revision of the intellectual content, besides approving the final version.

\section{Acknowledgments}

The authors wish to thank the principals of the ten schools from which the sample was selected and the students and staff that participated in the data collection process.

\section{References}

1. Blair SN, Kohl III HW, Paffenbarger Jr. RS, Clark DG, Cooper KH, Gibbons LW. Physical fitness and all-cause mortality: a prospective study of healthy men and women. JAMA 1989; 262:2395-401.

2. Paffenbarger Jr. RR, Hyde RT, Wing AL, Hsieh CC. Physical activity, all-cause mortality, and longevity of college alumni. N Engl J Med 1986; 314:605-13.

3. Dumith S. Physical activity in Brazil: a systematic review. Cad Saúde Pública 2009; 25 Suppl 3:S415-26.

4. U.S. Department of Health and Human Services. Physical activity and health: a report of the Surgeon General. Atlanta: U.S. Department of Health and Human Services/Centers for Disease Control and Prevention/National Center for Chronic Disease Prevention and Health Promotion; 1996.

5. Bernstein MS, Morabia A, Sloutskis D. Definition and prevalence of sedentarism in an urban population. Am J Public Health 1999; 89:29-33.

6. Martínez-González MA, Varo JJ, Santos JL, Irala J, Gibney M, Kearney J, et al. Prevalence of physical activity during leisure time in the European Union. Med Sci Sports Exer 2001; 33:1142-6.

7. Carvalhaes M, Moura EC, Monteiro CA. Prevalência de fatores de risco para doenças crônicas: inquérito populacional mediante entrevistas telefônicas em Botucatu, São Paulo, 2004. Rev Bras Epidemiol 2008; 11:14-23.
8. Monteiro CA, Moura EC, Jaime PC, Lucca A, Florindo AA, Figueiredo IC, et al. Monitoramento de fatores de risco para doenças crônicas por entrevistas telefônicas. Rev Saúde Pública 2005; 39:47-57.

9. Silva MA, Rivera IR, Ferraz MR, Pinheiro AJ, Alves SW, Moura AA, et al. Prevalência de fatores de risco cardiovascular em crianças e adolescentes da rede de ensino da cidade de Maceió. Arq Bras Cardiol 2005; 84:387-92.

10. Azevedo MR, Araújo CL, Silva MC, Hallal PC. Tracking of physical activity from adolescence to adulthood: a population-based study. Rev Saúde Pública 2007; 41:69-75.

11. Raustorp A, Svenson K, Perlinger T. Tracking of pedometer-determined physical activity: a 5 -year follow-up study of adolescents in Sweden. Pediatr Exerc Sci 2007; 19:228-38.

12. Anderssen N, Jacobs Jr. DR, Sidney S, Bild DE, Stemfeld B, Slaterry ML, et al. Change and secular trends in physical activity patterns in young adults: a seven-year longitudinal follow-up in the Coronary Artery Risk Development in Young Adults Study (CARDIA). Am J Epidemiol 1996; 143:351-62.

13. Nelson MC, Neumark-Stzainer D, Hannan PJ, Sirard JR, Story M. Longitudinal and secular trends in physical activity and sedentary behavior during adolescence. Pediatrics 2006; 118:e1627-34. 
14. Silva DAS, Silva RJS. Padrão de atividade física no lazer e fatores associados em estudantes de Aracaju/SE. Rev Bras Ativ Fís Saúde 2008; 13:94-101.

15. Farias Junior JC. Prevalência e fatores de influencia para inatividade física em adolescentes. Rev Bras Ciênc Mov 2006; 14:63-70.

16. Hallal PC, Bertoldi AD, Gonçalves H, Victora CG. Prevalência de sedentarismo e fatores associados em adolescentes de 10-12 anos de idade. Cad Saúde Pública 2006; 22:1277-87.

17. Farias Júnior JC, Lopes AS, Mota J, Hallal PC. Prática de atividade física em adolescentes do Nordeste do Brasil. Rev Saúde Pública 2012; 46:505-15.

18. Oehlschlaeger MH, Pinheiro RT, Horta B, Gelatti C, San'tana P. Prevalence of sedentarism and its associated factors among urban adolescents. Rev Saúde Pública 2004; 38:157-63.

19. Crocker PR, Bailey DA, Faulkner RA, Kowalski KC, McGrath R. Measuring general levels of physical activity: preliminary evidence for the Physical Activity Questionnaire for Older Children. Med Sci Sports Exerc 1997; 29:1344-9.

20. Kowalski K, Crocker P, Faulkner R. Validation of the physical activity questionnaire for older children. Pediatr Exerc Sci 1997; 9:174-86.

21. Silva RCR, Malina RM. Nível de atividade física em adolescentes do Município de Niterói, Rio de Janeiro, Brasil. Cad Saúde Pública 2000; 16:1091-7.

22. Dumith S. Atividade física e sedentarismo: diferenciação e proposta de nomenclatura. Rev Bras Ativ Fís Saúde 2010; 15:253-4.

23. Associação Nacional de Empresas de Pesquisa. Critério de classificação econômica Brasil. São Paulo: Associação Nacional de Empresas de Pesquisa; 2011.

24. Alves JGB, Siqueira PP, Figueiroa JN. Overweight and physical inactivity in children living in favelas in metropolitan region of Recife, Brazil. J Pediatr (Rio J.) 2009; 85:67-71.

25. Rivera IR, Silva MA, Silva RD, Oliveira BA, Carvalho AC. Physical inactivity, TV-watching hours and body composition in children and adolescents. Arq Bras Cardiol 2010; 95:159-65.

26. Fermino RC, Rech CR, Hino AA, Rodriguez-Añez CR, Reis RS. Physical activity and associated factors in high-school adolescents in Southern Brazil. Rev Saúde Pública 2010; 44:986-95.

27. Tenório MCM, Barros MVG, Tassitano RM, Bezerra J, Tenório JM, Hallal PC. Atividade física e comportamento sedentário em adolescentes do ensino médio. Rev Bras Epidemiol 2010; 13:105-17.

28. Hallal PC, Knuth AG, Cruz DKA, Mendes MI, Malta DC. Prática de atividade física em adolescentes brasileiros. Ciênc Saúde Coletiva 2010; 15 Suppl 2:S3035-42.

29. World Health Organization. Young people's health in context. Health Behaviour in School-aged Children (HBSC) study: international report from the 2001/2002 survey. Geneva: World Health Organization; 2004.

30. Butcher K, Sallis JF, Mayer JA, Woodruff S. Correlates of physical activity guidelines compliance for adolescents in 100 U.S. cities. J Adolesc Health 2008; 42:360-8.
31. Babey SH, Brown ER, Hastert TA. Access to safe parks helps increase physical activity among Teenagers. Los Angeles: Center for Health Policy Research, University of California; 2005.

32. Lasheras L, Aznar S, Merino B, López EG. Factors associated with physical activity among Spanish youth through the national health survey. Prev Med 2001; 32:455-64

33. Prista A, Nhantumbo L, Saranga S, Lopes V, Maia J, Seabra A, et al. Physical activity assessed by accelerometry in rural African school-age children and adolescents. Pediatr Exerc Sci 2009; 21:384-99.

34. Shi Z, Lien N, Kumar BN, Holmboe-Ottesen G. Physical activity and associated socio-demographic factors among school adolescents in Jiangsu Province, China. Prev Med 2006; 43:218-21.

35. Moraes ACF, Fernandes CAM, Elias RGM, Nakashima ATA, Reichert FF, Falcão MC. Prevalência de inatividade física e fatores associados em adolescentes. Rev Assoc Med Bras 2009; 55:523-8.

36. Oliver M, Schofield GM, Schluter PJ. Parental influences on preschoolers' objectively assessed physical activity. J Sci Med Sport 2010; 13:303-309.

37. Karppanen AK, Ahonen SM, Tammelin T, Vanhala M, Korpelainen R. Physical activity and fitness in 8-year-old overweight and normal weight children and their parents. Int J Circumpolar Health 2012; $71: 17621$

38. Raphaelli CO, Azevedo MR, Hallal PC. Associação entre comportamentos de risco à saúde de pais e adolescentes em escolares de zona rural de um município do Sul do Brasil. Cad Saúde Pública $2011 ; 27: 2429-40$

39. Silva ICM, Knuth AG, Amorim TEC, Kremer MM, Rombaldi AJ, Hallal PC, et al. Atividade física de pais e filhos: um estudo de base populacional. Rev Bras Educ Fís Esp 2008; 22:257-63.

40. Siegel SR, Malina RM, Reyes MEP, Barahona EEC, Cumming SP. Correlates of physical activity and inactivity in urban Mexican Youth. Am J Hum Biol 2011; 23:686-92.

41. Sanz-Azure E, Ponce-de-León-Elizondo A, Valdemoros-San-Emeterio MA. Parental predictors of physical inactivity in Spanish adolescents. J Sports Sci Med 2012; 11:95-101.

42. Santos CM, Wanderley Júnior RS, Barros SSH, Farias Júnior JC, Barros MVG. Prevalência e fatores associados à inatividade física nos deslocamentos para a escola em adolescentes. Cad Saúde Pública 2010; 26:1419-30.

43. Gordon-Larsen P, McMurray RG, Popkin BM. Determinants of adolescent physical activity and inactivity patterns. Pediatrics 2000; 105:1-8.

44. Perry CK, Saelens BE, Thompson B. Intrapersonal, behavioral, and environmental factors associated with meeting recommended physical activity among rural Latino youth. Pediatr Exerc Sci 2011; 23:521-36.

Submitted on 31/May/2012

Final version resubmitted on 20/Mar/2013

Approved on 03/Jun/2013 